\title{
Robust Trajectory Design for Ballistic Landings on Dimorphos.
}

\author{
Iosto Fodde* and Jinglang Feng ${ }^{\dagger}$ and Massimiliano Vasile \\ University of Strathclyde, 75 Montrose Street, Glasgow G1 1XJ, United Kingdom
}

\begin{abstract}
ESA's Hera mission is planning to land a probe on the surface of the secondary body of the Didymos binary asteroid system, called Dimorphos, using a ballistic landing strategy. Ballistic landings can be sensitive to uncertainties in the deployment of the lander and the environment models. Thus, the design of the ballistic landing trajectory needs to take into consideration both the minimization of the touchdown velocity and the robustness of the trajectory against uncertainties. In this research, a landing trajectory design algorithm that accomplishes both these tasks is developed. This is done using the Generalised Intrusive Polynomial Algebra (GIPA) method to propagate the uncertainties through the dynamical system. A novel addition to GIPA is introduced, which is able to obtain the probability distribution of the spacecraft state over time. The landing trajectory design method is tested using several different landing area sizes and locations. It is found that there is a large difference between the found minimal touchdown velocities between landing locations. Furthermore, a larger allowed landing area results in more deployment states being allowed, at the expense of higher touchdown velocities. The algorithm developed here thus allows for the design of robust ballistic landing trajectories for landing on small bodies like Dimorphos.
\end{abstract}

\section{Nomenclature}

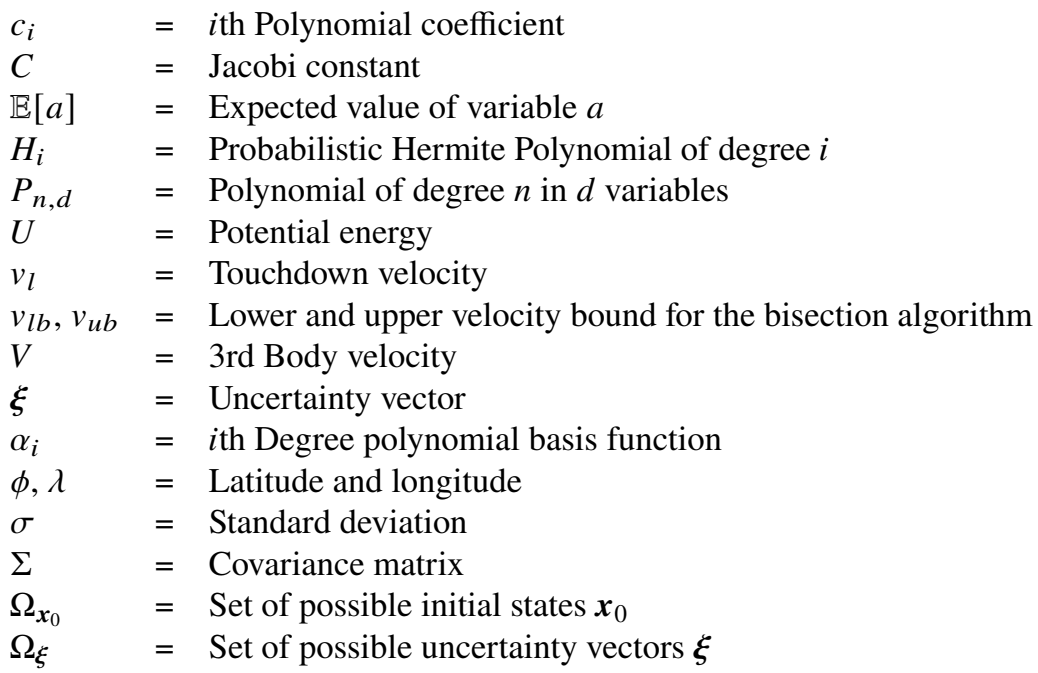

\section{Introduction}

A combined NASA and ESA multi-spacecraft mission (called DART and Hera respectively) plans to send a set of spacecraft to binary asteroid (65803) Didymos. The objectives of these missions and collaboration are threefold [1]: to investigate the possible planetary defence strategy of a kinetic impactor, increase our knowledge on small solar system bodies, and improve the technology needed for future asteroid missions. The DART mission itself was launched in November 2021 and will perform the kinetic impact with the secondary body of the system, Dimorphos. ESA's contribution, Hera, plans to arrive at the asteroid approximately 5 years later to investigate the effect of DART's kinetic

\footnotetext{
*PhD Researcher, Mechanical and Aerospace Engineering, iosto.fodde@ @strath.ac.uk.

†Lecturer and Chancellor's Fellow, Mechanical and Aerospace Engineering, jinglang.feng@strath.ac.uk.

†Professor, Mechanical and Aerospace Engineering, massimiliano.vasile@strath.ac.uk.
} 
impact in more detail and perform additional scientific observations. As part of the Hera mission, two CubeSats are planned to be released from the mothership once arrived, and orbit the system closely to perform detailed scientific observations. For one of the CubeSats, Juventas [2], the plan is to land on to the surface of Dimorphos at the end of the mission lifetime. During the descent of Juventas, the on board camera's will take pictures of the surface of Dimorphos and possibly the impact site of DART. The gravimeter, IMU, and gyro's are further used to capture the landing to get a better understanding of the composition of Dimorphos.

Previous landings on minor solar system bodies were attempted by Hayabusa [3], Rosetta [4], Hayabusa 2 [5], and OSIRIS-REx [6]. The main difficulties with the design of a landing system are the non-linear dynamics and the uncertainties in the physical properties of the system. Specifically, for a landing on the secondary body in a binary system, the procedure can be complicated due to the low gravitational forces and the large influence of the primary body. A ballistic landing, i.e. no position and velocity control maneuvers during the landing, can be preferential as it removes the need for a dedicated guidance, navigation, and control system. This significantly reduces the complexity and mass of the lander, which is a key limitation of the $6 \mathrm{U}$ Juventas CubeSat. However, this does require a robust landing trajectory design as the lander will not have the capabilities to counter any unforeseen dynamical disturbances and modelling errors. The dynamics of a spacecraft in a binary system is complicated and usually modeled using the circular restricted three body problem (CR3BP) model. Despite its complexities, solutions of the CR3BP can present favourable conditions for ballistic landings. This was shown in [7], where it was discussed how the stable and unstable manifolds around the co-linear Lagrange points of the CR3BP, specifcally $L_{2}$, can be used as minimal energy gateways to the surface of the secondary when deploying a lander around the $L_{2}$ point. The robustness of this strategy was further investigated in [8], where a deployment success rate was determined when considering off-nominal conditions for deployment from $L_{2}$. The work done in [9] looked further at the change of the invariant manifolds for close observation and landing when considering non-spherical bodies and different rotational rates. It was found that the elongation of the body and the rotational rate of the asteroid have a significant impact on the amount of trajectories being able to land on the surface. A novel method to determine minimal touchdown velocity landing trajectories that are deployed further from the binary system was developed in [10]. This method allows for targeting specific landing locations on the body and generates trajectories where the deployment can happen further away from the body. The investigation for ballistic landing trajectories specifically on the secondary of the Didymos system has seen an increase in interest in recent years for the contribution to the Hera mission, see e.g. [11] and [12].

In this work, the previous algorithm developed in [10] is expanded to meet the robustness requirements of ballistic landing trajectory design. Due to the fact that the observation of asteroids includes large errors, and that the deployment mechanism, being either a spring release or a impulsive maneuver, can result in off-nominal initial states, it is important to include the effect of these uncertainties in the design of landing trajectories. Previous research (e.g. [8] and [12]) performed the trajectory design initially using only a nominal model of the system and only afterwards investigated the effect of uncertainties. In this work, these two analyses are combined to estimate the landing trajectory that has minimal touchdown velocity for a specific landing area, given all the uncertainties present in the system. To accomplish this, an uncertainty propagation technique is required that is more accurate than the linearized covariance analyses (as the uncertainties might be too large) and more efficient than the conventional Monte Carlo analysis, while being able to create an approximation of the dynamical system. A widely used method in spaceflight mechanics is the polynomial algebra technique, which creates a polynomial approximation of the initial uncertainty set and propagates this set using an algebra constructed over the space of polynomials to obtain an approximation of the dynamics. This method was first developed using an algebra over Taylor polynomials, and was called differential algebra (DA) [13], or Jet Transport (JT) [14]. This was further expanded to a Chebyshev basis to make use of its greater approximation characteristics [15], and then generalized for any desired polynomial basis by [16]. This general method is called Generalised Intrusive Polynomial Algebra (GIPA), and will be used in this work. Another contribution of this paper is an addition to the GIPA technique, which allows the statistical moments to be obtained efficiently from the resulting polynomial approximation.

This paper is organized as follows. In section III] the dynamics of the binary asteroid system Didymos are discussed. Following this, in section IV]GIPA is explained in more detail, including a novel method to obtain an approximation of the probability distribution along the whole trajectory. Section $\nabla$ will explain further how GIPA is used to find robust ballistic landing trajectories. The results of this algorithm are then finally discussed in section $\mathrm{VI}$ and a conclusion will be given in VII 
Table 1 Relevant physical parameters of the Didymos system, taken from [17].

\begin{tabular}{ll}
\hline \hline & \\
System mass & $5.28( \pm 0.54) \cdot 10^{11} \mathrm{~kg}$ \\
Mass ratio & $0.0093 \pm 0.0013$ \\
Didymos Diameter & $780 \mathrm{~m} \pm 3 \mathrm{~m}$ \\
Didymos Rotational Period & $2.26 \mathrm{~h} \pm 0.0001 \mathrm{~h}$ \\
Dimorphos Diameter & $164 \mathrm{~m} \pm 18 \mathrm{~m}$ \\
Dimorphos Orbital Period & $12 \mathrm{~h}$ \\
Body separation distance & $1.19 \mathrm{~km}$ \\
\hline \hline
\end{tabular}

\section{Dynamical Modelling}

The binary asteroid system (68503) Didymos consists of two bodies, the primary body called Didymos and the secondary body Dimorphos. Some of the relevant physical properties of the binary system are given in table 1 . Both Didymos and Dimorphos rotate around a single axis pointing orthogonal to the binary's orbital plane, where Dimorphos is tidally locked with Didymos, meaning that the same face of Dimorphos is always facing towards Didymos. In this work, a synodic reference frame that rotates together with the orbit of Dimorphos is considered. The origin of this frame is located in the barycentre of the system, the x-axis points towards Dimorphos, the z-axis to the orbit normal, and the y-axis completes the right-handed frame (see figure 1 ).

To determine the equation of motion of a spacecraft in the Didymos system, the circular restricted three-body problem (CR3BP) is used, where it is assumed that the mass of the third body (in this case the spacecraft) is negligible compared to the masses of the other bodies. Furthermore, it is also assumed that the binary orbit is constrained to a circular orbit, which is in close agreement with observations of the Didymos system [18]. The equations of motion of the CR3BP are defined as [19]:

$$
\begin{aligned}
\ddot{x}-2 \dot{y} & =\frac{\partial U}{\partial x}, \\
\ddot{y}+2 \dot{x} & =\frac{\partial U}{\partial y}, \\
\ddot{z} & =\frac{\partial U}{\partial z} .
\end{aligned}
$$

Here, the mass parameter $\mu=m_{2} /\left(m_{1}+m_{2}\right)$, the body separation distance $R$, and the time constant $1 / n$ (where $n$ is the mean motion of Dimorphos) were used to obtain dimensionless parameters and simplify the equations to only include $\mu$ and the dimensionless coordinates $x, y$, and $z$. The potential function $U$ consists of both the gravitational potential and the rotational part of the equations of motion. For the goals of this research, the spherical gravity models are used for simplicity and computational efficiency. The potential is thus given as follows:

$$
U=\frac{1}{2}\left(x^{2}+y^{2}\right)+\frac{1-\mu}{r_{1}}+\frac{\mu}{r_{2}},
$$

where $r_{1}$ and $r_{2}$ represent the distance from the spacecraft to the primary and secondary, respectively.

The CR3BP allows an integral of motion called Jacobi's constant, given by [19]:

$$
C=2 U-V^{2},
$$

where $V$ is the velocity of the 3rd body. This variable can be seen as an energy measure, where lower values correspond to higher energy spacecraft trajectories. Constant values of $C=2 U$ give surfaces where the velocity of the spacecraft is zero and are called zero-velocity surface (ZVS). The ZVS restricts the motion of the spacecraft to certain regions in space, as is shown by the black lines in figure 1 . For high values of $C$, the ZVS blocks transport between the region around the two bodies, and between the inner regions and the region outside of the system (figure 1a). As the energy of trajectories increases (decreasing value of $C$ ), the region near the first Lagrange point $L_{1}$ opens up to allow transport between the two bodies (figure 1b). Increasing the energy even further opens up the $L_{2}$ point, which then 


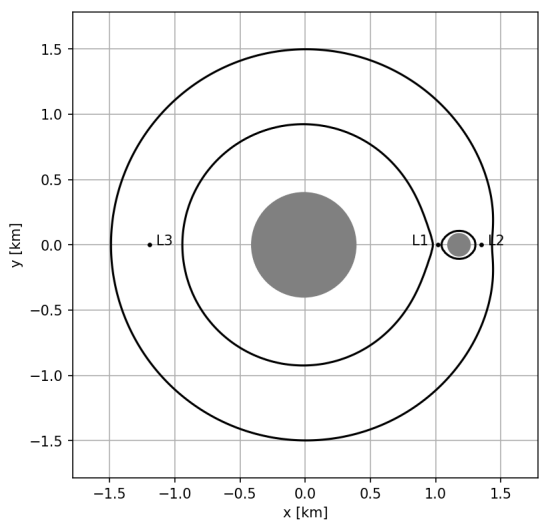

(a) $C=3.17$.

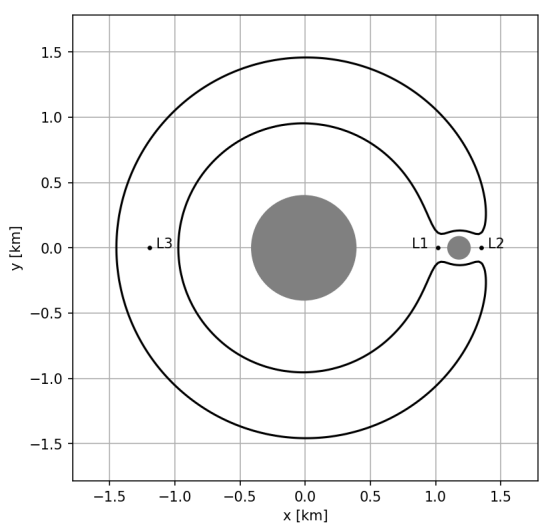

(c) $C=3.13$.

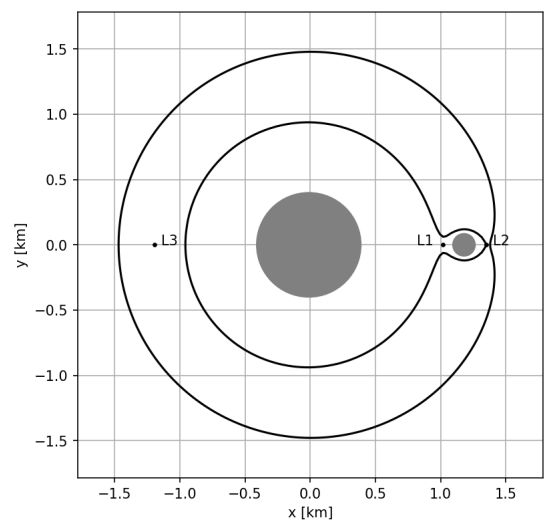

(b) $C=3.15$

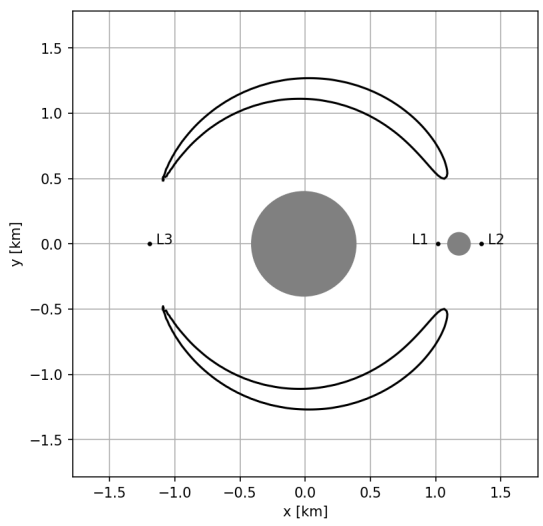

(d) $C=3.01$

Fig. 1 The ZVS plotted for three different Jacobi constant values.

allows for the spacecraft to enter the inner region of the binary system (figure $1 \mathrm{c}$. Then, as $C$ decreases more, the ZVS opens at the $L_{3}$ point (figure 1d). For a given trajectory to be able to land on the surface of the secondary, $C$ must have a sufficiently low value such that the ZVS is not blocking access to the surface through the $L_{2}$ point [7]. Then the stable and unstable manifolds of the $L_{2}$ point can present feasible trajectories for landing.

\section{Uncertainty Propagation}

The design of a certain landing trajectory is based on a nominal environment model and a single initial state vector $\boldsymbol{x}$. However, imperfections in the insertion strategy and uncertainties stemming from Earth-based observations of the system result in off-nominal conditions when performing the landing maneuver. The effect of these uncertainties on the landing trajectories can be investigated by using uncertainty propagation (UP) techniques. In this research, the GIPA method is used, which will be described in detail as follows.

\section{A. Generalised Intrusive Polynomial Algebra}

Consider a compact set $\Omega_{\xi}$, which represents all possible values of the uncertainty vector $\boldsymbol{\xi}$, containing all the uncertain variables. A specific realisation of $\boldsymbol{\xi}$ at time $t_{0}$ gives the initial state $\boldsymbol{x}\left(t_{0}\right)=\boldsymbol{x}_{0}$. The set of all possible initial states from realisations of $\boldsymbol{\xi}$ is given by:

$$
\Omega_{x_{0}}=\left\{\boldsymbol{x}\left(t_{0}, \boldsymbol{\xi}\right) \mid \forall \boldsymbol{\xi} \in \Omega_{\xi}\right\} .
$$

The goal is then to obtain $F_{t}(\xi)$ which propagates this set through the nonlinear dynamical system $f(x, t)$, given as 
follows:

$$
F_{t}(\xi)=\left\{\boldsymbol{x}(t, \boldsymbol{\xi}) \mid \boldsymbol{x}(t, \boldsymbol{\xi})=\boldsymbol{x}_{0}+\int_{t_{0}}^{t} \boldsymbol{f}(\boldsymbol{\xi}, \tau) d \tau \forall \boldsymbol{\xi} \in \Omega_{\xi}\right\} .
$$

A perfect representation of this set would require an infinite number of samples to be propagated, thus it is required to approximate this set. If $\boldsymbol{x}_{t}$ is continuous in $\boldsymbol{\xi}$ and the set is compact, $F_{t}(\boldsymbol{\xi})$ can be approximated using a polynomial:

$$
\tilde{F}_{t}(\boldsymbol{\xi})=P_{n, d}(\boldsymbol{\xi})=\sum_{i=0}^{\mathcal{N}} c_{i}(t) \alpha_{i}(\boldsymbol{\xi}),
$$

where $\alpha_{i}(\boldsymbol{\xi})$ are a set of multivariate polynomial basis functions, $c_{i}(t)$ are the corresponding coefficients, and $\mathcal{N}=\left(\begin{array}{c}n+d \\ d\end{array}\right)$ is the number of terms of the polynomial, where $n$ is the degree of the polynomial and $d$ is the number of variables.

In this work, the generalised intrusive polynomial algebra (GIPA) is used to obtain Eq. (8), which was developed in [16]. GIPA starts by selecting a specific polynomial basis $\alpha$ and approximate the initial set $\Omega_{x_{0}}$ using the polynomial constructed from the basis functions $\alpha$. Then to obtain the set at future times, the space of polynomials $\mathcal{P}_{n, d}(\alpha)$ needs to be equipped with a set of elementary arithmetic operations, which enables the use of (numerical) integrators to propagate the set through the dynamical system. Instead of defining a specific set of operations for each different polynomial basis, a change of basis is performed to a monomial basis $\phi$. The main advantages of this is that it significantly reduces the computational cost and makes sure that only one set of elementary arithmetic operations needs to be implemented [16]. A set of elementary functions, $\otimes$, corresponding to similar operations in a floating point algebra, $\oplus \in\{+,-, \cdot, /\}$, is combined with the function space $\mathcal{P}_{n, d}(\phi)$ to create an algebra. Then, given any two functions $f_{a}$ and $f_{b}$, and their polynomial approximations $F_{a}$ and $F_{b}$, the same operations between the two functions in the floating point algebra can be represented in the polynomial algebra:

$$
f_{a} \oplus f_{b} \sim F_{A} \otimes F_{B}
$$

Besides the set of elementary operations, a set of elementary functions $h(y)$, e.g. $\{1 / y, \sin (y), \exp (y), \log (y)$, etc. $\}$, need to be represented in the algebra as well. This can be done using the composition operator as follows:

$$
h(\boldsymbol{f}(\boldsymbol{x})) \sim H(y) \circ F(\boldsymbol{x}),
$$

where $H(y)$ is the univariate polynomial representation of $h(y)$ and $F(\boldsymbol{x})$ the polynomial algebra representation of the multivariate set $\boldsymbol{f}(\boldsymbol{x})$. The composition operator is defined as follows:

$$
\circ: \mathcal{P}_{n, 1}(\phi) \times \mathcal{P}_{n, d}(\phi) \rightarrow \mathcal{P}_{n, d}(\phi) .
$$

The method by which $H(y)$ is obtained for the different elementary function depends on the specific chosen polynomial basis and is of importance to the efficiency and accuracy of the method. For a Taylor polynomial basis, a truncated Maclaurin series is used for the approximation. This approximation can be analytically obtained, which increases its computational efficiency, however it loses its accuracy away from the expansion point and doesn't handle discontinuities well. For a Chebyshev basis, an order 100 Chebyshev interpolation is used, which is more accurate over the whole range of the expansion, however being less computationally efficient compared to the Maclaurin series. For the Chebyshev interpolation an estimation of the range over which the expansion is taken is needed. This range estimation can either be done by taking the magnitude of the polynomial coefficients, or by taking a set of uniformly distributed random samples from the polynomial and saving the maximum and minimum values. It was shown in [16] that the coefficient based method results in an overestimation of the range, which decreases the accuracy. The sampling based method does not suffer from this drawback, however it is less efficient then the coefficient based method. In this work both methods will be considered.

The basic process of using GIPA for uncertainty propagation is then as follows. First a polynomial approximation is taken over the uncertain variables at the initial time using a specifically chosen basis. This basis is then transformed to the monomial basis to be able to use the general set of elementary operations, while using the elementary function approximation methods of the chosen polynomial basis. Using the desired numerical integrator, e.g. Runge-Kutta 4, the set can be propagated to the final desired time, using the algebra to replace each elementary operation and elementary function in the integrator and dynamical system. The convenience of this method is that no significant changes need to be made to the implementation of the methods that are normally used to propagate a single trajectory, 
as only the elementary operations and functions need to be replaced, which can be done in several different coding languages by overloading the operations. In this research, the SMART-UQ package is used for this [20], which contains implementations of the algebra for various different polynomial bases.

\section{B. Statistical Analysis}

The sets discussed in section IV.A do not contain any statistical information. These sets are all a representation of all possible states that can occur due to the uncertainties present in the system, where the uncertainties are only characterized by an interval. To obtain information on the likelihood of certain events happening or states being realized, a set of samples need to be generated from the desired probability distribution and propagated using the polynomial. This is more efficient than other methods like the Monte Carlo method to obtain information on the time evolution of the distribution, but can still be computationally complex when a large number of samples are required to be drawn due to large sets or complex dynamics.

In this work, a novel method is introduced that obtains the desired statistical information from the results of the GIPA method. This technique is based on the Polynomial Chaos Expansion (PCE) method, which represents a random variable in terms of a set of polynomials [21]. The specific polynomials are chosen such that they are orthogonal with respect to a weight function that represents the joint probability density function (pdf) of the random variable. In [22], an overview is given of the specific type of polynomials that represent different distributions. In this work, only the Hermite polynomial is considered, which represents a Gaussian pdf.

In PCE, the coefficients of the polynomial, representing the distribution of the random variable at a certain point in time, can be found by using several different methods, like Galerkin projection or least squares estimation [23]. In the method discussed here, the coefficients are obtained by performing a basis transformation from the GIPA obtained polynomial (in a monomial basis) to the desired basis representing a specific pdf, in this case the Hermite basis $(\eta)$, represented as follows:

$$
P_{n, d}(\xi)=\sum_{\boldsymbol{i},|\boldsymbol{i}| \leq n} c_{\phi, \boldsymbol{i}}(t) \phi_{\boldsymbol{i}}(\boldsymbol{\xi})=\sum_{\boldsymbol{i},|\boldsymbol{i}| \leq n} c_{\eta, \boldsymbol{i}}(t) H_{\boldsymbol{i}}(\boldsymbol{\xi}) .
$$

where $H$ is the probabilistic Hermite polynomial and the index vector $\boldsymbol{i}$ is defined in [24]. This basis transformation is performed using the following relationship [25]:

$$
x^{n}=n ! \sum_{m=0}^{n / 2} \frac{1}{2^{m} m !(n-2 m) !} H_{n-2 m}(x) .
$$

From the polynomial in Hermite basis, given in Eq. (12), the orthogonality condition of the basis can be exploited to obtain an analytical expressions for the first and second moment of the distribution [23]. The solution for the mean can be obtained using the fact that $\mathbb{E}\left[H_{0}\right]=1$ and $\mathbb{E}\left[H_{i}\right]=0, \forall i \neq 0$, which results in:

$$
\mathbb{E}\left[F_{t}(\boldsymbol{\xi})\right] \approx \mathbb{E}\left[\tilde{F}_{t}(\boldsymbol{\xi})\right]=\int_{\Omega_{\xi}}\left(\sum_{i,|\boldsymbol{i}| \leq n} c_{\eta, \boldsymbol{i}}(t) H_{\boldsymbol{i}}(\boldsymbol{\xi})\right) \rho(\boldsymbol{\xi}) d \xi=c_{\eta, 0},
$$

The covariance matrix can be obtained similarly, using the orthonormality of $H_{i}$ with the probability measure $\rho$ :

$$
\left.\Sigma(t)=\int_{\Omega_{\boldsymbol{\xi}}}\left(\sum_{\boldsymbol{i},|\boldsymbol{i}| \neq 0} c_{\eta, \boldsymbol{i}}(t) H_{\boldsymbol{i}}(\boldsymbol{\xi})\right)\right) \cdot\left(\sum_{\boldsymbol{i},|\boldsymbol{i}| \neq 0} c_{\eta, \boldsymbol{i}}(t) H_{\boldsymbol{i}}(\boldsymbol{\xi})\right)^{T} \rho(\boldsymbol{\xi}) d \xi=\sum_{\boldsymbol{i},|\boldsymbol{i}| \neq 0} c_{\eta, \boldsymbol{i}}(t) \cdot c_{\eta, \boldsymbol{i}}^{T}(t) .
$$

Thus, using the uncertain dynamical model obtained by the GIPA method, and the statistical moments obtained from the basis transformation explained here, an analysis can be made of both the dynamics and statistics of the ballistic landing trajectory.

\section{Robust Landing Trajectory Design}

In [10], a bisection algorithm to find ballistic landing trajectories was discussed. An upper and lower bound for the touchdown velocity was selected based on the velocity needed to open up the $L_{2}$ point. Then, for a given latitude and longitude on Dimorphos, a trajectory is generated by selecting a velocity in the middle of the velocity bounds and 
propagating the motion backwards in time from the surface to a safe deployment point away from the system. Based on the resulting trajectory, a set of criteria is used to determine if the currently selected velocity should be the new upper or lower bound for the next trajectory. This process is repeated until the velocity interval is sufficiently small. This analysis was extended to include uncertainties in the GNC system and Dimorphos density in [26], where a Monte Carlo analysis was performed to determine the robustness of certain trajectories previously found. However, this only tests trajectories for their robustness, instead of finding specific trajectories that are more robust. In this work, the goal is to find trajectories that are specifically robust against both uncertainties in the initial conditions and in the environment models.

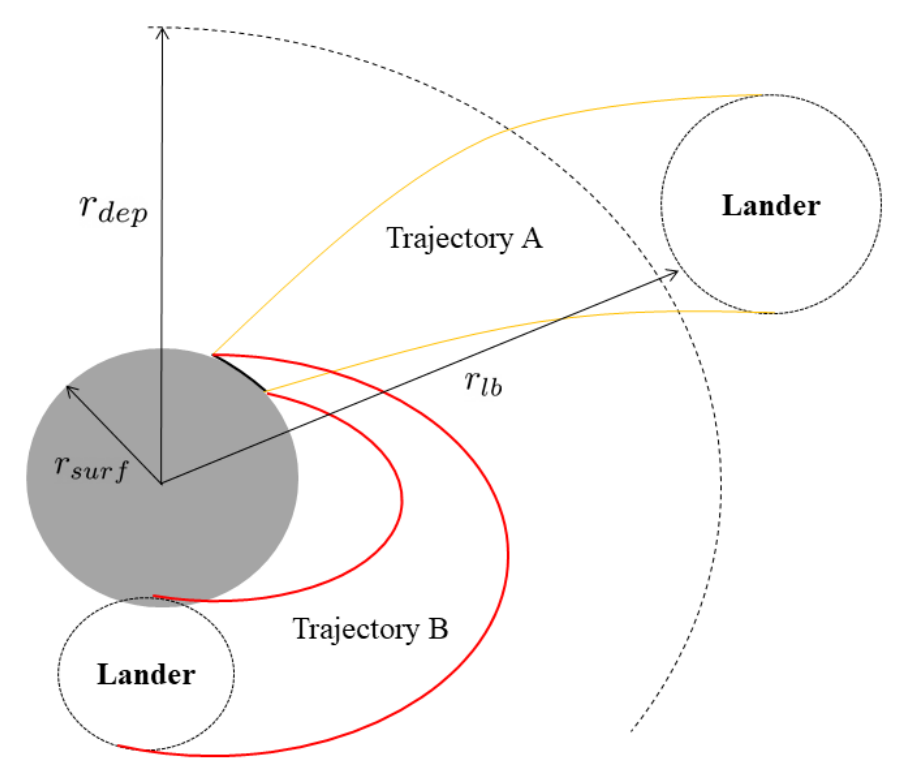

Fig. 2 Diagram explaining the robust trajectory design algorithm. The gray ellipse is Dimorphos and the dark area on its border is the desired landing area. The large dashed ellipses are a representation of the uncertain position set of the lander.

The algorithm defined here aims to find a minimum touchdown velocity $v_{l}$ for a given area in which the spacecraft is allowed to land. This is done by first selecting a nominal landing latitude $\phi$ and longitude $\lambda$, and defining the allowed uncertainty in both these parameters $\left(\sigma_{\phi}, \sigma_{\lambda}\right)$. Next to this, the estimated uncertainty in both the gravitational parameter of Didymos and Dimorphos is also used as an input $\left(\sigma_{\mu_{p}}, \sigma_{\mu_{s}}\right)$. The lower bound for the touchdown velocity is then taken to be the minimum velocity needed to open up the ZVS around the $L_{2}$ point, for which an estimate is given by [10]:

$$
v_{l b}=\sqrt{x^{2}+y^{2}+2 \frac{1-\mu}{r_{1}}+2 \frac{\mu}{r_{2}}-C_{L_{2}}},
$$

where $C_{L_{2}}$ is the estimated value of the Jacobi constant at the $L_{2}$ point. For Dimorphos, the initial $v_{l b}$ is around $3.2 \mathrm{~cm} / \mathrm{s}$. The upper bound for the velocity $v_{u b}$ is then taken to be a sufficiently large multiple of the lower bound. The initial guess for the touchdown velocity $v_{l}$ is taken as the middle point of the interval spanned by $v_{l b}$ and $v_{u b}$. Using the GIPA method, the uncertainties are then converted into a polynomial approximation and propagated backwards in time using the algebra, see figure 2 During the propagation, the distance between the surface of Dimorphos and the lower bound of the state uncertainty, $r_{l b}$, is checked. If this lower bound is below the surface of Dimorphos (surface radius given by the sphere $r_{\text {sur } f}$ ), then the propagation is stopped and the current touchdown velocity becomes the lower bound for the next propagation (see trajectory B in figure 2). If the lower bound reaches a pre-defined safe deployment distance from Dimorphos, $r_{d e p}$, the propagation stops and the current touchdown velocity becomes the following touchdown velocity upper bound (see trajectory A in figure 2). If after a maximum of 12 hours both of these 
events haven't happened, the propagation is stopped and the lower bound will be changed to the current touchdown velocity. This process is continued until the lower and upper bound of the touchdown velocity are sufficiently close (less then the value of TOL, which is taken as $1 e-10$ ). The algorithm then saves $v_{l}$, the lower bound of the Jacobi constant $C_{l b}$, and the standard deviation of the position and velocity, $\sigma_{r}$ and $\sigma_{v}$ respectively, obtained using the method explained in section IV.B. A summary of this process can be found in algorithm 1 . The result is a set of deployment conditions that will be able to land in the desired area of landing with a minimal touchdown velocity.

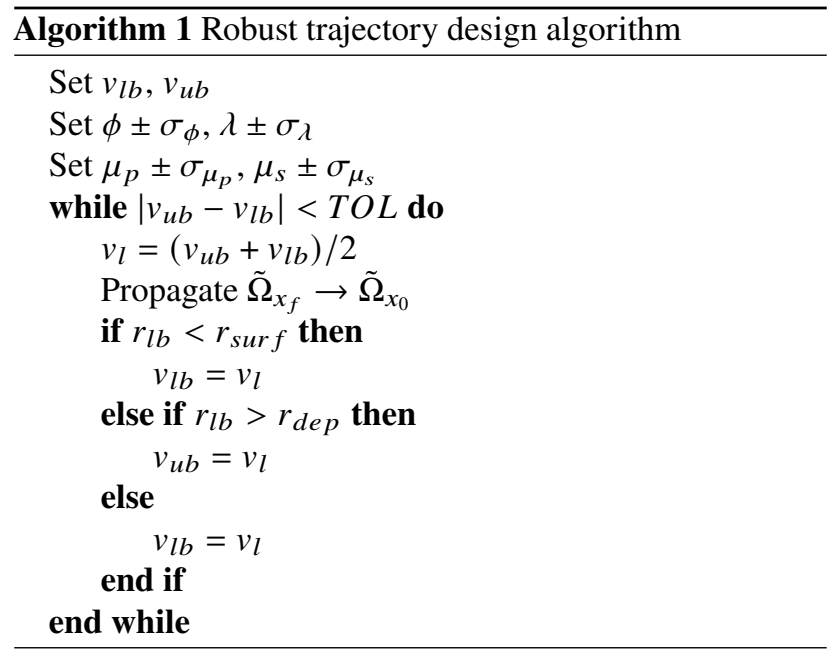

\section{Results}

In this section, two different landing locations are considered, and two different landing area sizes will be investigated. For both the gravitational parameter of Didymos and Dimorphos an uncertainty of 10 percent is taken into account. To test the influence of the different polynomial bases for the expansion of the dynamics, three different setups are considered here: Taylor basis, Chebyshev basis with coefficient based range estimation (CRE), and Chebyshev basis with sample based range estimation (SRE). For the SRE, only for the approximation of the elementary functions $h(y)$ will the sampling be used. For estimating the lower bound of the state to determine if the set has reached the deployment range, the CRE method is used to make sure that the whole set has reached deployment as the CRE overestimates the range compared to the underestimation of the SRE. For all bases, after experimentation a polynomial degree of 4 was determined to be optimal. The deployment position is chosen to be from a region around $1500 \mathrm{~m}$ away from the center of Didymos, to correspond to a scenario where the CubeSat is orbiting the system at a safe distance before performing a landing deployment maneuver.

Three different test cases will be analysed: $\phi=\lambda=0^{\circ} ; \sigma_{\phi}=\sigma_{\lambda}=10^{\circ}$ (case 1), $\phi=70^{\circ} ; \lambda=0^{\circ} ; \sigma_{\phi}=\sigma_{\lambda}=10^{\circ}$ (case 2), and $\phi=\lambda=0^{\circ} ; \sigma_{\phi}=\sigma_{\lambda}=25^{\circ}$ (case 3).

\section{A. Case 1}

For case 1, a nominal landing location of $\phi=\lambda=0^{\circ}$ is chosen, with a landing area uncertainty size of 10 degrees in both coordinates. The found touchdown velocity $v_{l}$ together with the lower bound of the Jacobi constant for the set $C_{l b}$ (thus the highest energy trajectory of the set), the standard deviation for the deployment position $\sigma_{p}$ and velocity $\sigma_{v}$ (found using the Hermite basis transformation), and the flight time $t_{l}$ are shown in Table 2, A set of 250 sample trajectories taken from the resulting polynomials are also shown in figure 3 As this is the landing location facing the $L_{2}$ point, it is expected that the minimum velocity is close to the minimum velocity needed to open up this point, mentioned in section V] All three basis setups show similar results where the minimum found touchdown velocity is between 4.2 and $4.5 \mathrm{~cm} / \mathrm{s}$, which is around $1 \mathrm{~cm} / \mathrm{s}$ higher then this $L_{2}$ minimum velocity. This extra velocity comes both from that an uncertain landing area is considered and not a single point, and that the spacecraft is required to reach a deployment position beyond at $r_{d e p}$ beyond the $L_{2}$ point. The main difference in the bases results is that a higher standard deviation is found for the deployment position and velocity of the Chebyshev SRE setup. 


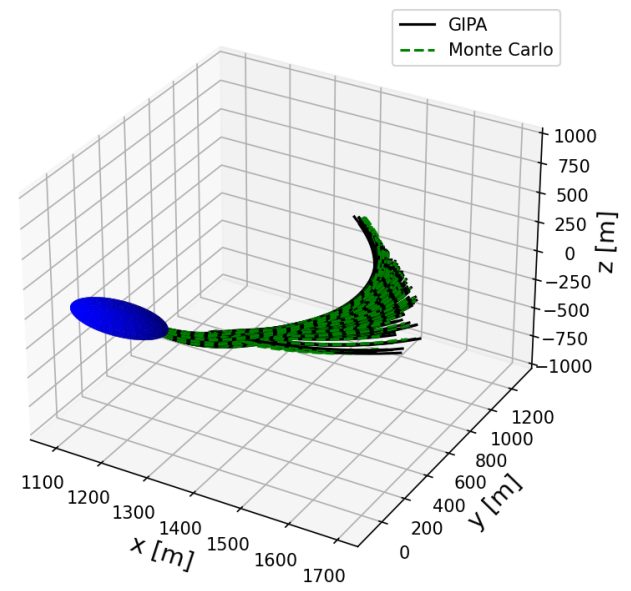

(a) Taylor

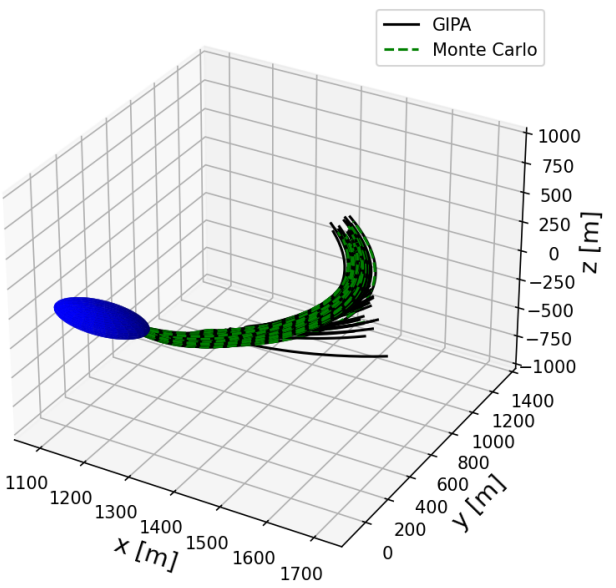

(b) Chebyshev CRE

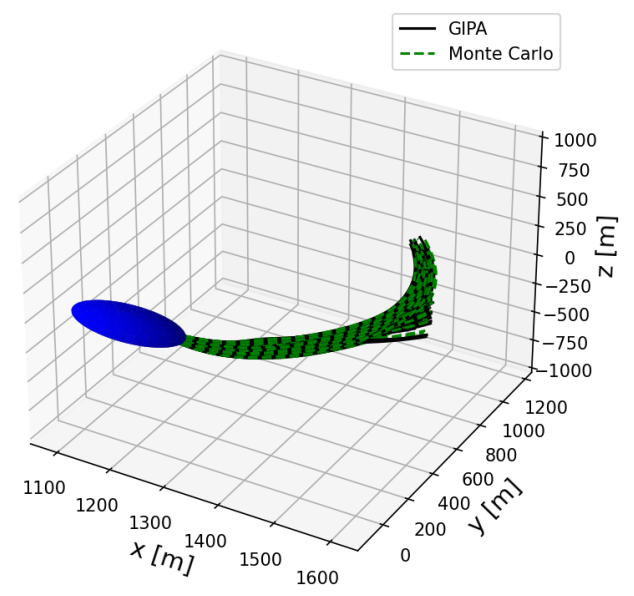

(c) Chebyshev SRE

Fig. 3 Samples taken from the resulting polynomials for Case 1 compared with a Monte Carlo simulation. 


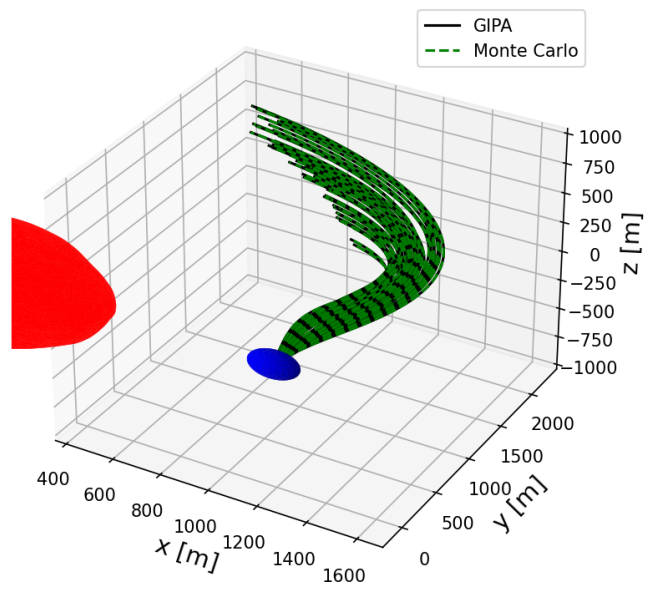

(a) Taylor

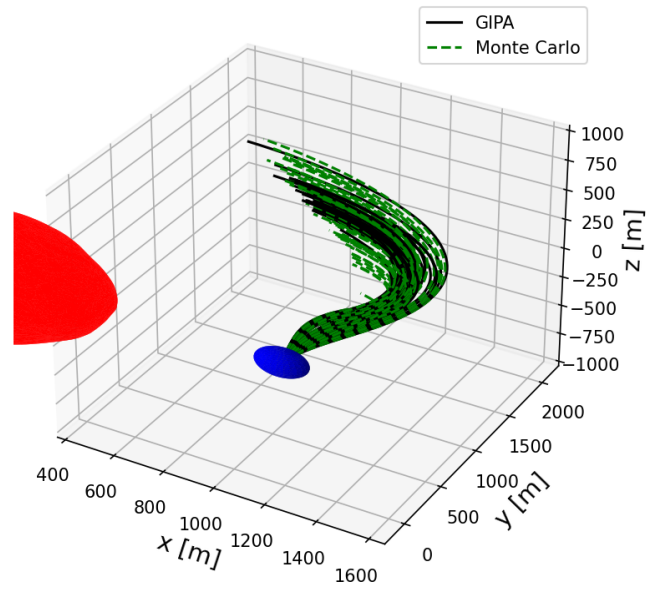

(b) Chebyshev CRE

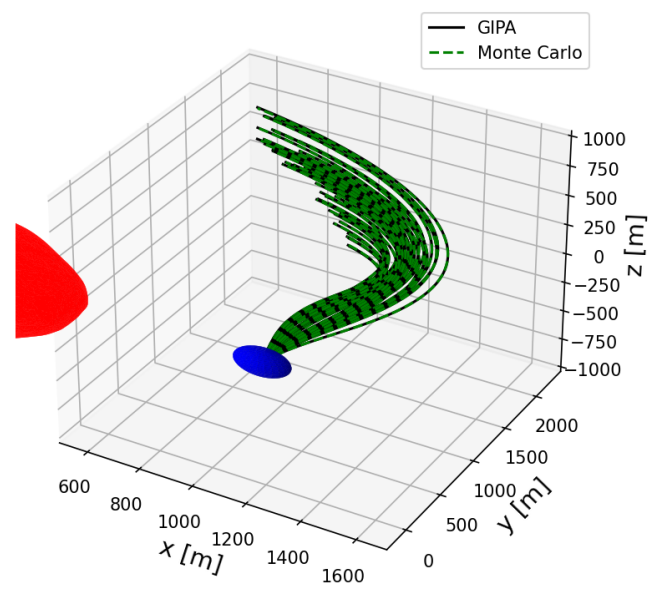

(c) Chebyshev SRE

Fig. 4 Samples taken from the resulting polynomials for Case 2 compared with a Monte Carlo simulation. 


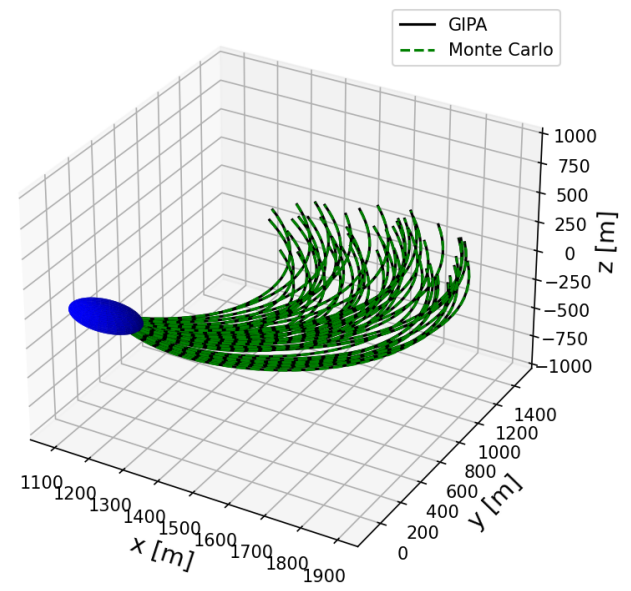

(a) Taylor

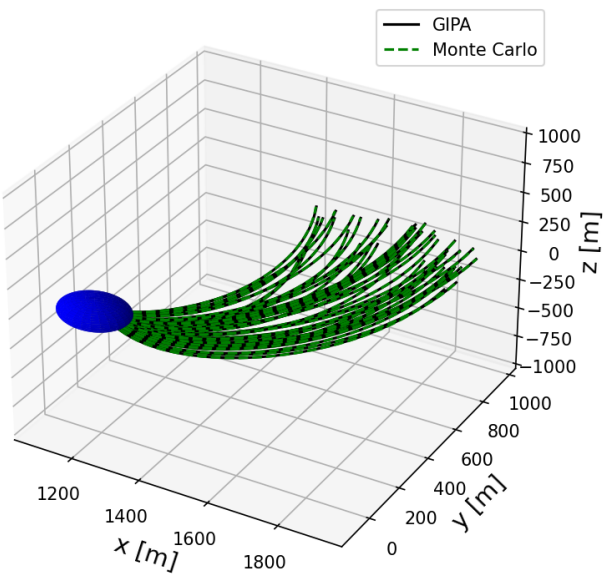

(b) Chebyshev CRE

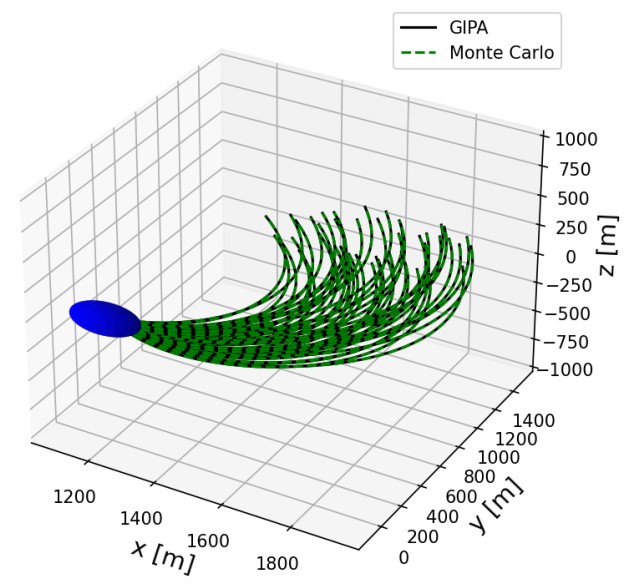

(c) Chebyshev SRE

Fig. 5 Samples taken from the resulting polynomials for Case 3 compared with a Monte Carlo simulation. 


\section{B. Case 2}

For case 2, where a higher latitude landing position of $70^{\circ}$ is taken, but the uncertain landing area remains the same, it can be seen from table 2 that the results are significantly different compared to those of case 1 . For this higher latitude case, a touchdown velocity of between 8.3 and $9.55 \mathrm{~cm} / \mathrm{s}$ is found, thus showing the need for higher energy trajectories and thus more of the ZVS opening up to allow these trajectories to land here. As will be shown in section VI.D the reason that the Chebyshev CRE shows different results is because it is much less accurate due to the overestimation of the range happening when the spacecraft is close to the body for a longer flight times. From figure 4, it can also be observed that due to the higher latitude, the spread of the trajectories in the z-direction is much larger then that of case 1. This shows as well in table 2, where a higher position and velocity standard deviation is found on average for case 2 compared to case 1. This allows for a wider range of deployment states to be allowed to land in this region.

\section{Case 3}

Finally, for case 3 , the same landing location as case 1 is taken, with a higher value for the uncertain landing area of $25^{\circ}$. It can be immediately seen from table 2, what the trade-off is for this case. Namely, this case allows for a higher standard deviation in both the position and velocity, thus allowing a larger range of deployment states. This can be seen more clearly when comparing figure 3 with figure 5 . However, this also results in a significantly higher touchdown velocity of around $7.8 \mathrm{~cm} / \mathrm{s}$. This higher touchdown velocity also results in a generally higher velocity along the trajectory, resulting in a relatively short duration of the trajectories of between 3 - 4 hours, compared to the other trajectories from case 1 and 2, which are all $5-6.5$ hours.

Table 2 Trajectory results for different polynomial bases for the different test cases.

\begin{tabular}{c||c|c|c|c|c}
\hline \hline & $v_{l}[\mathrm{~cm} / \mathrm{s}]$ & $C_{l b}[-]$ & $\sigma_{r_{d}}[\mathrm{~m}]$ & $\sigma_{v_{d}}[\mathrm{~m} / \mathrm{s}]$ & $t_{l}[\mathrm{~h}]$ \\
\hline Case 1: & \multicolumn{5}{|l}{} \\
\hline Taylor & 4.20 & 3.08 & 117.56 & 2.84 & 5.42 \\
Chebyshev CRE & 4.47 & 2.96 & 121.99 & 2.58 & 5.08 \\
Chebyshev SRE & 4.31 & 3.09 & 150.21 & 4.07 & 5.42 \\
\hline \multicolumn{5}{||}{} & \multicolumn{5}{|l}{} \\
Case 2: & 9.51 & 2.77 & 249.15 & 4.01 & 6.17 \\
\hline Taylor & 8.31 & 2.13 & 166.25 & 3.61 & 6.5 \\
Chebyshev CRE & 9.55 & 2.94 & 239.55 & 3.99 & 5.67 \\
Chebyshev SRE & 9.55 & & \\
\hline & \multicolumn{5}{|l}{} \\
Case 3: & 7.83 & 2.92 & 284.77 & 5.72 & 3.75 \\
\hline Taylor & 7.73 & 2.72 & 207.11 & 5.19 & 3 \\
Chebyshev CRE & 7.82 & 2.95 & 276.57 & 5.79 & 3.67 \\
Chebyshev SRE & 73
\end{tabular}

\section{Accuracy}

To verify the accuracy of the GIPA generated trajectories, a comparison is made with a Monte Carlo (MC) simulation of 1000 samples, sampled first using a uniform distribution bounded by the uncertainties and then using a normal distribution to verify the estimation of the statistical moments using the Hermite basis. The root mean square error (RMSE) is used as a performance metric for the GIPA accuracy, and is calculated as follows:

$$
R M S E=\sqrt{\frac{1}{N_{S}} \sum_{i=1}^{N_{s}}\left(\hat{x}_{i}-x_{i}\right)^{2}},
$$

where $N_{s}$ is the number of samples, $\hat{x_{i}}$ is the GIPA calculated state, and $x$ is the MC state at the same point in time. 
Figure 6 shows the accuracy for each case with the different lines indicating the different bases. As the uncertainty set is propagated backwards in time, the propagation starts at the time of landing and stops at time 0 , corresponding to the deployment time. For case 1, it can be seen that at first, both the Chebyshev bases are more accurate compared to the Taylor basis, however for the CRE setup the overestimation grows over time and thus after three hours the Taylor basis becomes more accurate. For the SRE setup, this doesn't happen and it remains more accurate compared to the Taylor basis. For case 2, a much larger difference can be found between the different bases. In this case the Taylor basis remains accurate across the landing trajectory, whereas especially the CRE Chebyshev basis loses a lot of accuracy. This can be seen as well in figure 4 where the Monte Carlo and GIPA trajectory samples are shown. This happens as the trajectory here spends a longer time closer to the body, which increases the overestimation happening because of the CRE. Case 3 shows higher initial RMSE, which is caused by the larger uncertainty set size used. Due to the higher velocity and the shorter flight time, the variation of RMSE is smaller than for the other cases. As expected, for the most part the two Chebyshev bases are more accurate than the Taylor basis due to the large uncertainty size. But over time due to the increase in overestimation from the CRE and the underestimation from the SRE, the bases become similar in terms of accuracy.

To determine the accuracy of the statistical moments from the Hermite basis, a MC analysis using a set of normally distributed samples was used. The standard deviation of this distribution was taken to be the uncertainties from the uncertain landing area and the gravitational parameters. This does result in several samples being outside the landing ellipse. As in this section is only concerned with the accuracy of the found statistical moments, this is not a problem. Figures $7 \mathrm{a}$ and $7 \mathrm{c}$ shows the evolution of the mean and standard deviation over time of the Taylor basis results from case 2 compared with the MC values, and figures $7 \mathrm{~b}$ and $7 \mathrm{~d}$ show the distribution of the position and velocity at the deployment time. The results show that the found distribution from the Hermite polynomial closely matches the MC results. This corresponds with the RMSE found for this specific case and basis in figure 6 To determine the effect of a less accurate GIPA result on the estimated distribution, case 1 is taken as an example as well. The results for the Taylor basis from case 1 are shown in figure 8 . Here it can be seen that the higher RMSE translates to a lower accuracy of the estimated distribution, as the difference between the statistical moments is higher then for the previous example. Thus, if a more accurate value for the distribution is needed, a higher polynomial degree to reduce the RMSE will also increase the accuracy of the estimated probability distribution.

\section{Conclusion}

In this work, a novel algorithm was developed that can design sets of possible landing trajectories for a given landing area on the secondary of a binary asteroid system, given uncertainties in the gravitational models of the asteroids. This technique uses the uncertainty propagation method called GIPA to perform a backwards propagation of a set of states from the desired landing area, to a deployment region from where the lander can be deployed to the surface. Using a bisection algorithm, the minimal touchdown velocity is then found for the entire landing area. Furthermore, using a newly developed method that transforms the monomial basis of GIPA to a Hermite basis, the statistical moments of a normal distribution propagated along the trajectory can be found from the GIPA results.

This method was then applied to the case of the a CubeSat landing on the surface of Dimorphos. For similar sizes of landing areas, it was found that larger touchdown velocities can be expected for higher latitude landing locations. For larger landing area sizes, the deployment region is larger, allowing less stringent requirements for the impulsive maneuver to get the lander on the landing trajectory. However, this does come at the cost of increasing the minimal touchdown velocity for that area.

The work performed here shows a new algorithm for the development of robust landing trajectories. Future work could include non-spherical gravity models for the asteroids to increase the fidelity of the simulations. Furthermore, a larger survey of the surface of Dimorphos should be performed to get a better idea of where the more difficult locations to land are. Finally, this work only considers the first contact of the lander with the asteroid. A more detailed analysis of the bouncing motion after touchdown could improve the robustness of the trajectories produced significantly.

\section{Acknowledgments}

The authors would like to acknowledge the ESA OSIP program for partly sponsoring this research project. Furthermore, the authors are grateful to Jesus Gil-Fernandez for his help and advice on Hera and Juventas. 


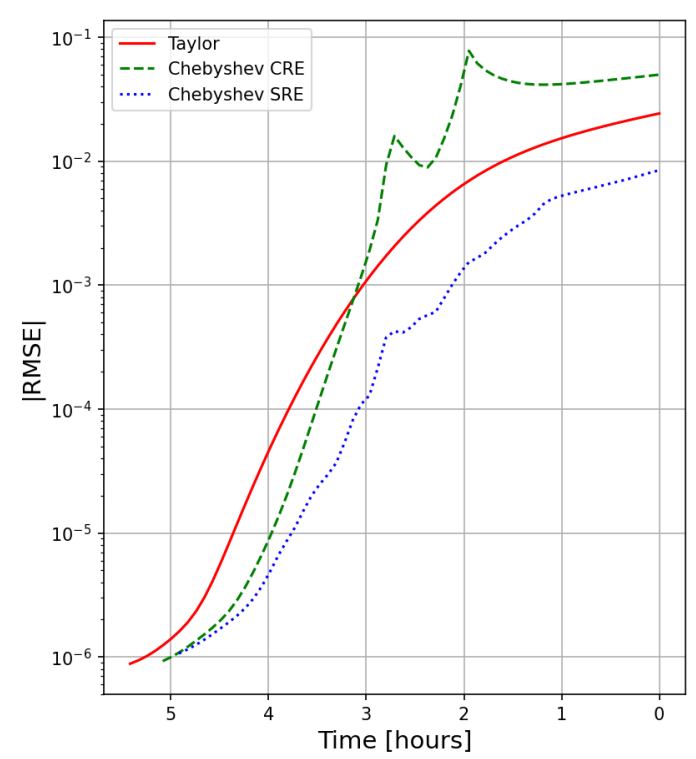

(a) Case 1

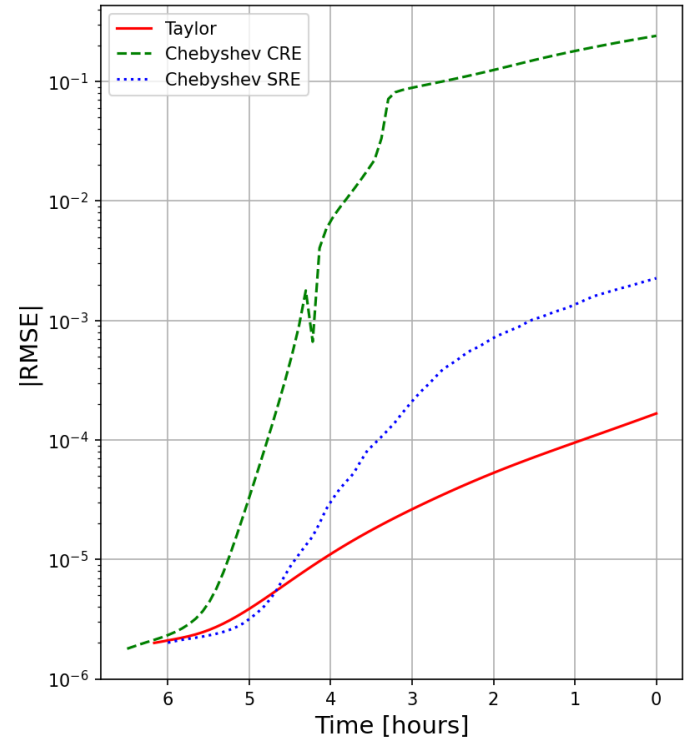

(b) Case 2

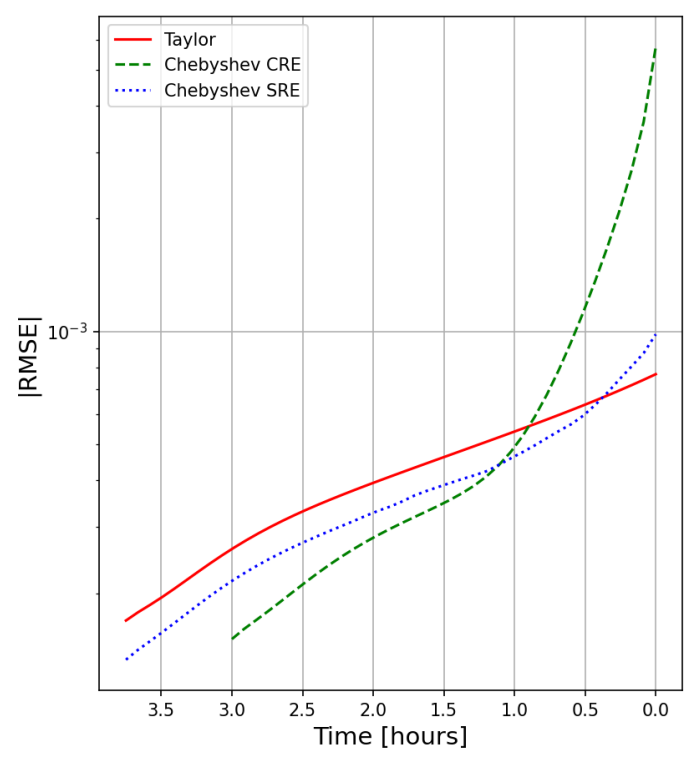

(c) Case 3

Fig. 6 Accuracy of the GIPA results compared with a Monte Carlo simulation. 


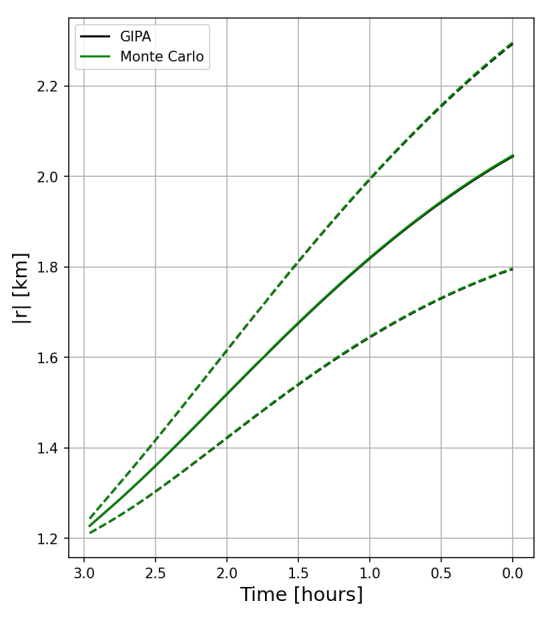

(a) Position time evolution.

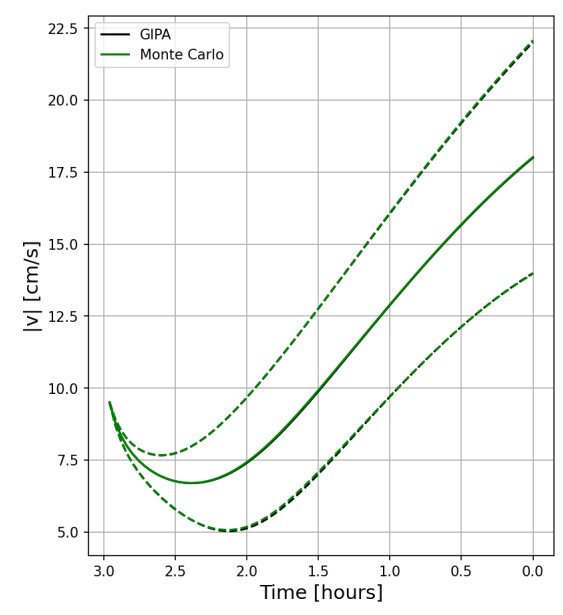

(c) Velocity time evolution.

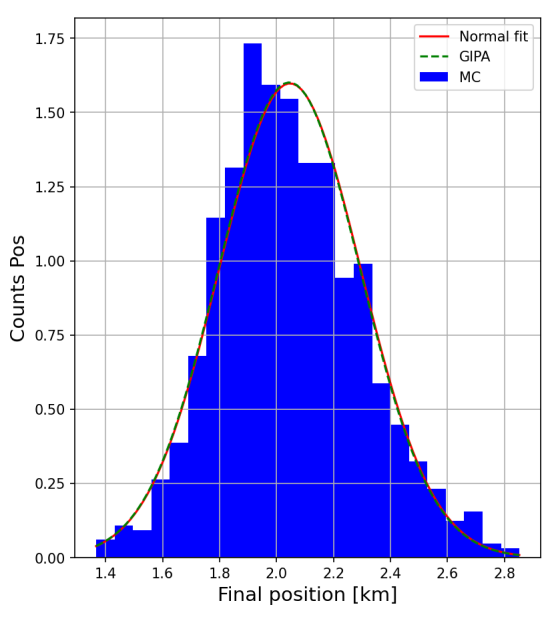

(b) Position deployment distribution.

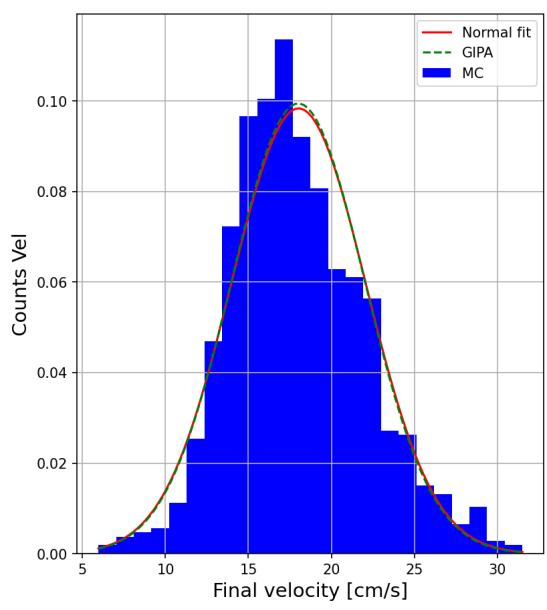

(d) Velocity deployment distribution.

Fig. 7 The probability distribution over time and specifically at the deployment time for the Taylor basis result of case 2. 


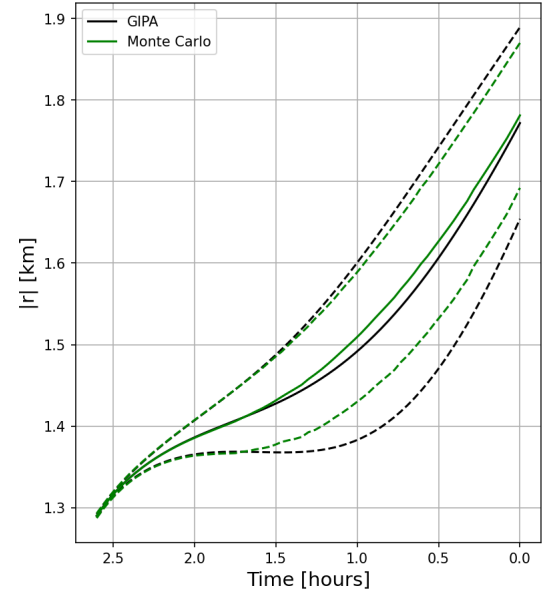

(a) Position time evolution.

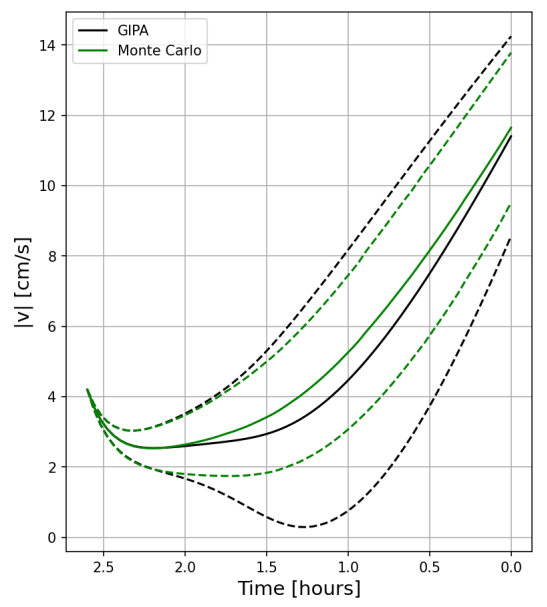

(c) Velocity time evolution

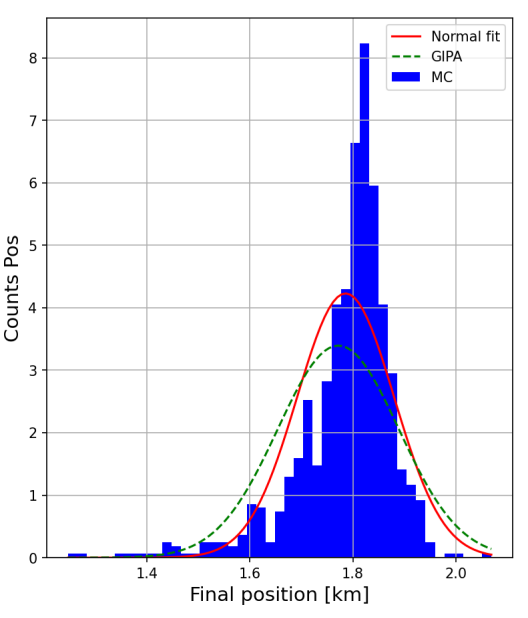

(b) Position deployment distribution.

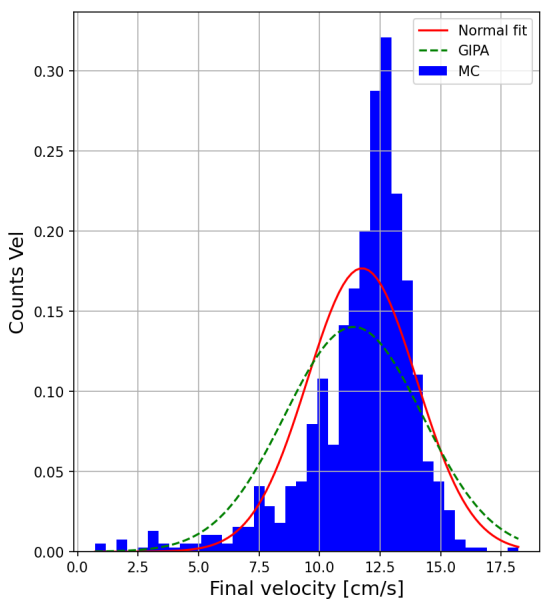

(d) Velocity deployment distribution.

Fig. 8 The probability distribution over time and specifically at the deployment time for the Taylor basis result of case 1 . 


\section{References}

[1] Michel, P., Küppers, M., Carnelli, I., Michel, P., Küppers, M., and Carnelli, I., "The science return of the ESA Hera mission to the binary asteroid Didymos," 43rd COSPAR Scientific Assembly, Vol. 43, 2021, p. 275. URL https://ui . adsabs.harvard edu/abs/2021cosp...43E.275M/abstract

[2] Goldberg, H., Karatekin, o., Ritter, B., Herique, A., Tortora, P., Prioroc, C., Gutierrez, B., Martino, P., and Carnelli, I., "The Juventas CubeSat in Support of ESA's Hera Mission to the Asteroid Didymos," Small Satellite Conference, 2019. URL https://digitalcommons.usu.edu/smallsat/2019/all2019/73.

[3] Kawaguchi, J., "Hayabusa, summary of guidance, navigation and control achievement in its proximity phase," Collection of Technical Papers - AIAA/AAS Astrodynamics Specialist Conference, 2006, Vol. 2, American Institute of Aeronautics and Astronautics Inc., 2006, pp. 1334-1341. https://doi.org/10.2514/6.2006-6533.

[4] Muñoz, P., Budnik, F., Companys, V., Godard, B., Casas, C. M., Morley, T., and Janarthanan, V., "Rosetta navigation during lander delivery phase and reconstruction of Philae descent trajectory and rebound," 25th International Symposium on Space Flight Dynamics, 2015.

[5] Tsuda, Y., Saiki, T., Terui, F., Nakazawa, S., Yoshikawa, M., and Watanabe, S. i., "Hayabusa2 mission status: Landing, roving and cratering on asteroid Ryugu," Acta Astronautica, Vol. 171, 2020, pp. 42-54. https://doi.org/10.1016/j.actaastro.2020.02.035

[6] Lauretta, D. S., Balram-Knutson, S. S., Beshore, E., Boynton, W. V., Drouet d'Aubigny, C., DellaGiustina, D. N., Enos, H. L., Golish, D. R., Hergenrother, C. W., Howell, E. S., Bennett, C. A., Morton, E. T., Nolan, M. C., Rizk, B., Roper, H. L., Bartels, A. E., Bos, B. J., Dworkin, J. P., Highsmith, D. E., Lorenz, D. A., Lim, L. F., Mink, R., Moreau, M. C., Nuth, J. A., Reuter, D. C., Simon, A. A., Bierhaus, E. B., Bryan, B. H., Ballouz, R., Barnouin, O. S., Binzel, R. P., Bottke, W. F., Hamilton, V. E., Walsh, K. J., Chesley, S. R., Christensen, P. R., Clark, B. E., Connolly, H. C., Crombie, M. K., Daly, M. G., Emery, J. P., McCoy, T. J., McMahon, J. W., Scheeres, D. J., Messenger, S., Nakamura-Messenger, K., Righter, K., and Sandford, S. A., "OSIRIS-REx: Sample Return from Asteroid (101955) Bennu,", 10 2017. https://doi.org/10.1007/s11214-017-0405-1. URL https://link-springer-com.proxy.lib.strath.ac.uk/article/10.1007/s11214-017-0405-1

[7] Tardivel, S., and Scheeres, D. J., "Ballistic deployment of science packages on binary asteroids," Journal of Guidance, Control, and Dynamics, Vol. 36, No. 3, 2013, pp. 700-709. https://doi.org/10.2514/1.59106/ASSET/IMAGES/LARGE/FIGURE10.JPEG. URL https://arc.aiaa.org/doi/abs/10.2514/1.59106.

[8] Tardivel, S., Michel, P., and Scheeres, D. J., "Deployment of a lander on the binary asteroid (175706) 1996 FG3, potential target of the european MarcoPolo-R sample return mission," Acta Astronautica, Vol. 89, 2013, pp. 60-70. https://doi.org/10.1016/J. ACTAASTRO.2013.03.007, URL https://www.sciencedirect.com/science/article/pii/S0094576513000799

[9] Herrera-Sucarrat, E., Palmer, P. L., and Roberts, R. M., “Asteroid Observation and Landing Trajectories Using Invariant Manifolds," Journal of Guidance Control and Dynamics, Vol. 37, No. 3, 2014, pp. 907-920. https://doi.org/10.2514/1.59594. URL https://openresearch.surrey.ac.uk/esploro/outputs/journalArticle/Asteroid-Observation-and-Landing-Trajectories-Using/ 99512229002346

[10] Çelik, O., and Sánchez, J. P., “Opportunities for ballistic soft landing in binary asteroids,” Journal of Guidance, Control, and Dynamics, Vol. 40, No. 6, 2017, pp. 1390-1402. https://doi.org/10.2514/1.G002181. URL www.aiaa.org/randp.

[11] Ferrari, F., and Lavagna, M., "Ballistic landing design on binary asteroids: The AIM case study," Advances in Space Research, Vol. 62, No. 8, 2018, pp. 2245-2260. https://doi.org/10.1016/J.ASR.2017.11.033

[12] Çelik, O., Sanchez, J. P., Karatekin, O., and Ritter, B., “A comparative reliability analysis of ballistic deployments on binary asteroids," Acta Astronautica, Vol. 156, 2019, pp. 308-316. https://doi.org/10.1016/J.ACTAASTRO.2018.03.020

[13] Valli, M., Armellin, R., Di Lizia, P., and Lavagna, M. R., "Nonlinear mapping of uncertainties in celestial mechanics," Journal of Guidance, Control, and Dynamics, Vol. 36, No. 1, 2013, pp. 48-63. https://doi.org/10.2514/1.58068 URL https://arc.aiaa.org/doi/abs/10.2514/1.58068.

[14] Pérez-Palau, D., Masdemont, J. J., and Gómez, G., “Tools to detect structures in dynamical systems using Jet Transport,” Celestial Mechanics and Dynamical Astronomy, Vol. 123, No. 3, 2015, pp. 239-262. https://doi.org/10.1007/s10569-015-9634-3. URL https://link.springer.com/article/10.1007/s10569-015-9634-3

[15] Riccardi, A., Tardioli, C., and Vasile, M., "An intrusive approach to uncertainty propagation in orbital mechanics based on tchebycheff polynomial algebra," Advances in the Astronautical Sciences, Vol. 156, Univelt Inc., 2016, pp. 707-722. 
[16] Vasile, M., Absil, C. O., and Riccardi, A., "Set propagation in dynamical systems with generalised polynomial algebra and its computational complexity," Communications in Nonlinear Science and Numerical Simulation, Vol. 75, 2019, pp. $22-49$. https://doi.org/10.1016/j.cnsns.2019.03.019

[17] “Hera Didymos Reference Model Issue 5," Tech. rep., ESA, 2020.

[18] Naidu, S. P., Benner, L. A., Brozovic, M., Nolan, M. C., Ostro, S. J., Margot, J. L., Giorgini, J. D., Hirabayashi, T., Scheeres, D. J., Pravec, P., Scheirich, P., Magri, C., and Jao, J. S., "Radar observations and a physical model of binary near-Earth asteroid 65803 Didymos, target of the DART mission," Icarus, Vol. 348, 2020, p. 113777. https://doi.org/10.1016/j.icarus.2020.113777

[19] Wakker, K., Fundamentals of Astrodynamics, Institutional Repository, Delft University of Technology, 2015.

[20] Absil, C. O., Riccardi, A., Vasile, M., and Tardioli, C., "SMART-UQ: uncertainty quantification toolbox for generalised intrusive and non intrusive polynomial algebra," 6th International Conference on Astrodynamics Tools and Techniques, Darmstadt, Germany, 2016. URL/https://pureportal.strath.ac.uk/en/publications/smart-uq-uncertainty-quantification-toolboxfor-generalised-intru

[21] Wiener, N., “The Homogeneous Chaos," American Journal of Mathematics, Vol. 60, No. 4, 1938, p. 897. https://doi.org/10. $2307 / 2371268$

[22] Xiu, D., and Em Karniadakis, G., "The Wiener-Askey Polynomial Chaos for Stochastic Differential Equations," SIAM Journal on Scientific Computing, Vol. 24, No. 2, 2006, pp. 619-644. https://doi.org/10.1137/S1064827501387826

[23] Jones, B. A., Doostan, A., and Born, G. H., "Nonlinear propagation of orbit uncertainty using non-intrusive polynomial chaos," Journal of Guidance, Control, and Dynamics, Vol. 36, No. 2, 2013, pp. 430-444. https://doi.org/10.2514/1.57599. URL https://arc.aiaa.org/doi/abs/10.2514/1.57599.

[24] Giorgilli, A., and Sansottera, M., "Methods of algebraic manipulation in perturbation theory," Third La Plata International School on Astronomy and Geophysics., 2013, pp. 147-183. URL http://arxiv.org/abs/1303.7398.

[25] Olver, F., Lozier, D., Boisvert, R., and Clark, C., NIST Handbook of Mathematical Functions, Cambridge University Press, 2010.

[26] Çelik, O., Sanchez, J. P., Karatekin, O., and Ritter, B., “Analysis of natural landing trajectories for passive landers in binary asteroids : A case study for ( 65803 ) 1996GT Didymos," 5th Planetary Defence Conference, , No. May, 2017, pp. 1-16. URL http://dspace.lib.cranfield.ac.uk/handle/1826/12310. 